\title{
Diseño y validación del cuestionario percepción del profesor sobre la deserción universitaria (CDUp)
}

\section{Design and validation of the questionnaire on teacher perception of university dropout (CDUp)}

\section{(1) Psicoconente ISSN 0124-013}
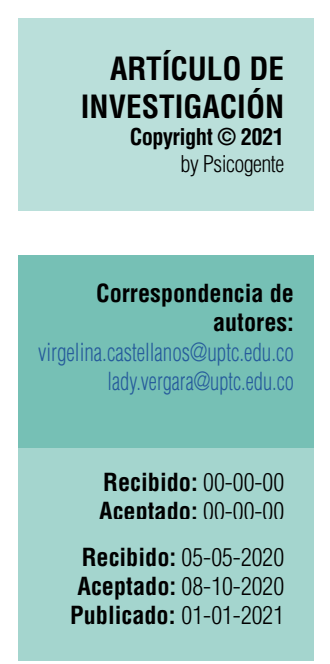

Resumen

Objetivo: Diseñar y validar el Cuestionario Percepción del profesor sobre la Deserción Universitaria (CDUp) basado en el modelo bioecológico de Bronfenbrenner

Método: En este estudio de tipo instrumental, participaron 464 profesores, el $74,1 \%$ eran mujeres (M edad $=40,05 ; D E=10,9)$ y el $25,9 \%$ eran hombres $(M$ edad $=40,09 ; D E=11,23)$, vinculados a una universidad pública $(n=249)$ y privada $(n=211)$ de la ciudad de Tunja (Colombia).

Resultados: El análisis Factorial Exploratorio (AFE) con método de extracción mínimos cuadrados y rotación promax, arrojó una estructura pentafactorial, explicando el 55,28 \% de la varianza. La consistencia interna del instrumento estimada con el coeficiente de Alfa de Cronbach $(\alpha=0,89-0,95)$ y el método de fiabilidad compuesta, Omega de McDonald $(\omega=0,89-0,95)$, reveló niveles de fiabilidad fuerte para todas las escalas: Intrapersonal, Adaptación personal, Redes de apoyo disfuncional, Interacción docente-estudiante, y Aspectos institucionales y empleabilidad.

Conclusión: El CDUp es un instrumento con óptimas propiedades psicométricas, el cual permite medir la percepción que tienen los profesores sobre los factores de riesgo asociados a la deserción universitaria.

Palabras clave: abandono escolar; deserción universitaria; cuestionario psicométrico; docentes universitarios; modelo ecológico de Bronfenbrenner; propiedades psicométricas.

\section{Abstract}

Objective: This paper aims to design and validate of the Questionnaire on Teacher Perception of University Dropout based on Bronfenbrenner's bioecological model.

Method: In this instrumental study, the University Dropout Questionnaire for use by teachers (CDUp, by its acronym in Spanish) was designed and validated in a sample of 464 teachers from a public and a private university of an intermediate city in Colombia. The construction of the questionnaire was carried out based on Bronfenbrenner's bioecological model.

Results: The Exploratory Factor Analysis (CFA) with least squares extraction method and promax rotation threw a five-factor theoretical structure, explaining $55,28 \%$ of the variance. The internal consistency of the instrument, estimated with Cronbach's coefficient alpha $(\alpha=0,89-0,95)$ and McDonald's coefficient omega $(\omega=0,87-0,95)$, revealed strong levels of reliability for all the scales: Intrapersonal, Personal Adaptation, Dysfunctional support networks, Teacher-student interaction, and Institutional aspects and employability.

Conclusion: It is concluded that the CDUp is an instrument that presents optimal psychometric properties, which allows the measurement of the perception of risk that teachers have of the students who abandon the university system.

Keywords: school dropout; university dropout; psychometric questionnaire; university teachers; Bronfrenbrenner's bioecological model; psychometric properties.

Cómo citar este artículo (APA):

Castellanos-Paez, V. \& Vergara Estupiñán, L.M. (2021). Diseño y validación del cuestionario percepción del profesor sobre la deserción universitaria (CDUp).Psicogente 24(45),(2021).1-20. https://doi.org/10.17081/psico.24.45.4128 


\section{INTRODUCCIÓN}

La deserción universitaria ha sido objeto de interés para las Instituciones de Educación Superior (IES) y para los Gobiernos de Colombia y América Latina, por las altas tasas de deserción reportadas que generan pérdidas millonarias y problemas para la salud física y mental de los jóvenes (Vries, Arenas, Romero \& Hernández, 2011). En América Latina, las tasas de deserción oscilan entre el $40 \%$ y el $75 \%$, siendo Bolivia, Nicaragua y Colombia los países que reportan más altas tasas de deserción por cohorte (Observatorio de Educación Superior de Medellín [ODES], 2017). En Colombia, se estima que el $42,36 \%$ de los estudiantes que comienzan un programa universitario lo abandonan, siendo los estudiantes de bajos ingresos financieros los más propensos a desertar, lo que implica, un truncamiento en la superación de la pobreza de las familias y en consecuencia, un problema de orden social y económico (Ferreyra, Avitabile, Botero, Haimovich, \& Urzúa, 2017).

La deserción universitaria ocurre cuando un estudiante no logra concluir su proyecto educativo y abandona la carrera o el sistema educativo, considerándose como desertor al estudiante que durante un año no reporta actividad académica (Giovagnoli, 2002). La deserción es un fenómeno multicausal que se explica por un conjunto de factores de tipo socioeconómico, académico, personal, familiar, pedagógico e institucional (González-Rodríguez, Vieira, \& Vidal, 2019; Román, 2013).

Diversos estudios han aportado evidencia sobre qué variables son relevantes en cada uno de estos factores. A nivel académico, se han encontrado variables como bajo rendimiento académico, los bajos resultados en las pruebas de desempeño, las altas tasas de reprobación (Barragán \& Patiño, 2013). A nivel individual, se resaltan como factores de riesgo, que el estudiante ingrese a la universidad sin las competencias necesarias, la dependencia económica de sí mismo, falta de esfuerzo, bajos niveles de motivación, métodos de estudio y estilos de aprendizaje no acordes con la carrera elegida (Gil, Antelm-Lanzat, Cacheiro-González, \& Pérez-Navío, 2018), dificultades en la capacidad de adaptación al sistema universitario que le exige autorregulación y autonomía (González, Montenegro, López, Munita, \& Collao, 2011; Warne, Svensson, Tirén, \& Wall, 2020) y las bajas expectativas con relación a la carrera elegida (Aparicio, 2012; Barragán \& Patiño, 2013).

A nivel familiar, Ottosen, Goll y Sørlie (2016) y Melo-Becerra, Ramos-Forero y Hernández-Santamaría (2017), identificaron como variables importantes, las presiones familiares y sociales, el desempleo de los padres, tener alguna 
calamidad, el bajo nivel educativo de los padres, los bajos ingresos familiares, experiencias negativas con adultos significativos, entre otras variables. A nivel pedagógico, se identifican como variables relevantes las metodologías de aprendizaje tradicionales, las dificultades en la comunicación profesor-estudiante, las deficiencias pedagógicas (i.e. falta de claridad expositiva, escasa motivación, mal uso de recursos didácticos, inadecuada evaluación) (Gil et al., 2018; Moreira-Mora, 2007; Quin, 2016; Román, 2013; Silvera, 2016).

Finalmente, a nivel institucional, González et al. (2011) y Melo-Becerra et al. (2017), señalan que la responsabilidad que un estudiante deserte recae en la gestión institucional y en los lineamientos de la política universitaria, debido principalmente a los aspectos organizativos, la infraestructura, la baja calidad del programa al que accede el estudiante, los recursos y el equipamiento de la institución universitaria.

Con la revisión metateórica realizada por Schimitt y Dos Santos (2016), a partir de la teoría bioecológica del desarrollo humano de Bronfenbrenner (1983, 1987, 2011) se integraron 33 variables reportadas en la literatura derivadas de distintos modelos explicativos basados en teorías sociológicas (Astin, 1985; Pascarella \& Terenzini, 2005) y en teorías psicológicas (Cabrera, Mejías, \& Fernández, 2015; Seidman, 1993). Desde esta postura teórica se asume la deserción como un fenómeno multicausal y multidimensional permeado por las diferentes condiciones sociales y ambientales que circundan al individuo. Las variables fueron categorizadas a nivel de la persona (e.g., características demográficas y familiares, condiciones financieras, relaciones con el trabajo y empleo, habilidades-competencias-conocimientos) el proceso (e.g., orientaciones vocacionales-profesionales, intenciones y metas educacionales), el contexto (e.g., factores ambientales, contexto socioeconómico, soporte familiar y parental, soporte institucional, feedback educacional y aspiraciones por la carrera / profesión) y el tiempo (e.g., expectativa temporal para la obtención de las metas académicas, tiempo dedicado a los estudios, expectativas futuras profesionales y de la carrera y experiencias previas). Este análisis a la luz de una postura bioecológica, permitió avanzar en la comprensión integrativa de las distintas variables y del reconocimiento de las condiciones socioambientales como determinantes de la decisión de desertar del estudiante.

Pese a que la evidencia empírica reportada converge en conceptualizar y explicar la deserción como un fenómeno multicausal y multidimensional e incluso hay una apuesta en proponer una mirada integrativa y ecológica del fenómeno de la deserción, los estudios y modelos explicativos surgen, en 
mayor medida, de estudios y modelos de deserción basados en la percepción y evaluación desde el estudiante, dejando de lado la percepción del profesor quien se configura como actor activo en los procesos de formación del estudiante.

Una visión ecológica de la deserción, supondría el análisis desde los distintos actores del sistema educativo (i.e., estudiante, profesor, directivos, familia) especialmente desde la perspectiva docente, quien hace parte de los sistemas de interacción más próximos del estudiante, quien identifica las fortalezas y dificultades académicas y media los procesos de aprendizaje y de ajuste al sistema universitario (González et al., 2011; Martínez-González \& Álvarez-Blanco, 2005; Márquez, Ortiz \& Rendón, 2008; Mejía et al., 2016; Olave-Arias, Rojas-García, \& Cisneros-Estupiñán, 2013). Asimismo, diferentes estudios muestran que el nivel de compromiso y la motivación académica del estudiante se ven influenciados por el tipo de interacciones entre profesor-estudiante, la metodología de clase y el sistema de evaluación, entre otros elementos. De manera que, la falta de empatía del profesor y una relación controladora que coarta la autonomía del estudiante, afecta el compromiso académico en sus dimensiones afectiva (e.g., sentimientos negativos hacia el aprendizaje), comportamental (e.g., baja participación) y cognitiva (falta de concentración y regulación de su aprendizaje, lo que se convierte en factores de riesgo para que un alumno abandone la universidad (Quin, Heerde \& Toumbourou, 2018; Rumberger \& Rotermund, 2012; Ryan \& Deci, 2000; Bal et al., 2018).

En esta línea, identificar las variables relacionadas con la deserción desde la perspectiva del profesor cobra relevancia, ya que, es él quien tiene contacto directo con los estudiantes, puede observar sus procesos académicos y detectar dificultades o fortalezas en los procesos de adaptación al sistema universitario (Márquez, Ortiz, \& Rendón, 2008). De esta forma, la percepción del profesor se convierte en un insumo clave para identificar cuáles son los principales factores asociados a la deserción y de esta manera generar modelos de evaluación temprana que permitan minimizar el riesgo de abandono del sistema universitario.

Estudios descriptivos realizados con profesores, han arrojado evidencia sobre los factores que ellos consideran relevantes a nivel del estudiante, la familia, el profesor y la institución (Palou \& Utges, 2012; Tejedor \& GarcíaValcárcel, 2007). Palou y Utges (2012) encontraron que los profesores tienen perspectivas diferenciadas de la deserción de acuerdo a sus teorías implícitas sobre las prácticas educativas. Algunos ejemplos relevantes de esta caracte- 
rización, permitieron identificar que profesores que consideran que un buen profesor es quien exige y debe establecer respeto, creen que los estudiantes desertan por problemas sociales y económicos y que es una cuestión externa a los profesores y a la Institución. Los profesores encuestados señalan que a los estudiantes les va mal, porque no saben razonar, lo que se les enseña desborda su capacidad de comprensión y se frustran con facilidad.

En el mismo estudio, los profesores que se ajustan al cumplimiento de las normas, afirman que las dificultades de los estudiantes de primer año se dan porque llegan inmaduros a la universidad, con poca claridad sobre su vocación, con baja formación de la Escuela a nivel de los conocimientos previos y falta de costumbre para asumir obligaciones. Profesores poco colaborativos y negativos, refieren que el manejo de la deserción es un asunto que compete a la Institución y es externa a ellos. Finalmente, profesores con perspectivas centradas más en mantener el interés y establecer una relación amigable con los estudiantes, consideran como factores relevantes de la deserción la falta de motivación, la empatía que ofrece el profesor considerando que su actuación es un factor decisivo en la retención de alumnos.

En la misma línea, Tejedor y García-Valcárcel (2007), en una muestra de 508 profesores, identificaron que consideran como variables asociadas al bajo rendimiento de los estudiantes variables de tipo individual como el escaso nivel de conocimientos previos para cursar las asignaturas, la falta de autocontrol, de autoexigencia y responsabilidad, así como, dejarse llevar por un ambiente más de diversión que de estudio en la Universidad. A nivel institucional, consideraron relevante el excesivo número de asignaturas que los estudiantes deben cursar, la falta de coordinación entre las materias y el clima poco motivador para el estudio. Frente a las variables del propio docente, consideraron como variable asociada la falta de estrategias para motivar a los estudiantes.

Finalmente, en el estudio de Díaz y Osuna (2017), los profesores consideraron como factores determinantes de la deserción escolar, el ausentismo escolar, la reprobación, las dificultades de aprendizaje, la falta de apoyo afectivo y educativo de la familia, la necesidad de trabajar y la influencia negativa de amistades. Estas variables son externas a su rol como docente, lo que implica, que los profesores consideran que el grado de compromiso y consecución de los logros académicos sean responsabilidad del estudiante. 
En suma, los estudios descriptivos que han examinado el fenómeno de la deserción, desde la perspectiva del profesor, arrojaron que para algunos docentes son relevantes variables de tipo individual, familiar, pedagógico e institucional y para otros, son relevantes variables externas a su rol como docente, priorizando variables del estudiante y del contexto familiar. La apuesta en este estudio, es aportar una herramienta objetiva, estructurada y validada para el contexto universitario, que permita identificar la percepción de los profesores considerando que la perspectiva del estudiante y del profesor puede ser complementarias y necesarias a la hora de explicar el fenómeno de la deserción y detectar el nivel de riesgo de forma oportuna.

Adicionalmente, resulta de gran interés para la psicología, diseñar un cuestionario que evalué la percepción del profesor sobre la deserción universitaria, desde un modelo ecológico, con el fin de aportar evidencia empírica en la explicación de este fenómeno. El modelo bioecológico de Bronfrenbrenner $(1983,1987,2011)$ es un modelo que conceptualiza la deserción como un fenómeno multivariado y multidimensional e integra el conjunto de variables de forma interrelacionada. En este modelo, se asume que el contexto universitario es un sistema, en el que las percepciones e interpretaciones que los estudiantes construyen sobre sí mismos y sobre los otros dependen de los procesos de interacción con sus ambientes próximos (e.g., familia, profesores, pares) y distales (e.g., políticas educativas, contexto laboral, la cultura).

De manera que, la decisión de desertar del estudiante se deriva de la interacción de múltiples factores que dificultan el nivel de ajuste o congruencia entre las expectativas y características personales (e.g., habilidades, capacidades, actitudes) y la calidad y el tipo de interacciones que se generan en los distintos sistemas de participación (la familia, escuela, pares) (Bronfenbrenner, 1995). En este sentido, el instrumento desarrollado evalúa distintas variables en cada subsistema. En el microsistema, se evalúan las características personales y adaptativas del estudiante que le permiten interactuar con su familia, pares y profesores. En el mesosistema se evalúa el tipo de interacciones que el estudiante establece con su familia, profesores y sus pares, y en el macrosistema, se evalúan los aspectos institucionales, empleabilidad, religión, entre otros aspectos, que afectan los comportamientos e interacciones del sujeto.

En esta línea, el objetivo de este estudio es presentar el diseño y validación del Cuestionario de Deserción Universitaria para profesores (CDUp) que 
apoye los procesos de tamizaje de deserción en las IES y se convierta en una herramienta de detección oportuna del riesgo de deserción universitaria. La información arrojada por el cuestionario permitiría a las IES comprender el fenómeno de la deserción y tomar decisiones a nivel de sus políticas, planes de acción que le permitan disminuir el riesgo de deserción de sus estudiantes.

\section{MÉTODO}

\subsection{Participantes}

Participaron 464 profesores de educación superior, el 74,1 \% eran mujeres $(\mathrm{M}$ edad $=40,05 ; \mathrm{DE}=10,9)$ y el 25,9\% eran hombres $(\mathrm{M}$ edad $=40,09 ; \mathrm{DE}=$ $11,23)$, vinculados a una universidad pública $(n=249)$ y privada $(n=211)$ de la ciudad de Tunja (Colombia), seleccionados por muestreo probabilístico, estratificado por tipo de universidad (pública y privada), y facultad, con un margen de error del $5 \%$. Los profesores se encontraban adscritos a las facultades de: Arquitectura, Diseño y Urbanismo (5,6\%), Educación (25,2 \%), Economía (3\%), Salud (18,8\%), Jurídica y Ciencias Sociales (3\%), Administrativa y Contable $(2,4 \%)$, Ciencias $(7,8 \%)$, Ciencias e Ingeniería (8,8 \%), Ingeniería $(18,3 \%)$ y Agronomía (4,8 \%). Los profesores fueron seleccionados de las bases de datos proporcionadas por cada facultad de las dos universidades ubicadas en una ciudad intermedia de Colombia. Como criterios de inclusión para la selección de los profesores se tuvo en cuenta que estuvieran vinculados a las dos universidades (i.e., privada y pública) y que hicieran parte de las facultades donde se reportara tasas de deserción académica superior al $35 \%$ y tasas de deserción inferiores al 35 \% (según reportes). El criterio de exclusión fue no consentir la participación en el estudio.

\subsection{Instrumentos}

\subsubsection{Cuestionario de Deserción Universitaria para profesores CDUp}

Es un instrumento de autoreporte para profesores universitarios que mide la percepción de los profesores sobre los factores de riesgos de la deserción universitaria. El CDUp, basado en el modelo bioecológico de Bronfenbrenner $(1983,1987,2011)$, se conforma por 59 ítems distribuidos en cinco escalas diferenciadas en tres niveles del sistema universitario: nivel micro, meso y macro, que permiten identificar los factores de riesgo de por qué un estudiante decide abandonar el sistema universitario desde la percepción del profesor. Para la construcción del instrumento se tuvo en cuenta la revisión teórica sobre deserción universitaria y estudios realizados con docentes. Este cuestionario se aplica de forma individual en formato 
impreso o digital, a los docentes universitarios, a quienes se les solicita que según su percepción valore de 0 a $4(0=$ desacuerdo -4 = acuerdo total) el nivel de relevancia que le otorga al riesgo asociado a la deserción de los estudiantes tanto por razones académicas como no académicas. Las puntuaciones directas de cada escala se ubican en tres percentiles que indican qué tanto riesgo de deserción representa para el docente cada uno de los cinco factores: sin riesgo, mediano riesgo y alto riesgo (ver Tabla 1). De esta forma, el profesor desde su percepción establece el nivel de riesgo a partir de las incongruencias que percibe en el estudiante y los diferentes entornos donde interactúa de acuerdo a su experiencia, teniendo en cuenta que el docente desde su quehacer se ve involucrado en las transformaciones y desarrollo de sus estudiantes.

En el microsistema se identifican factores individuales a nivel cognitivo, motivacional y vocacional del estudiante que le permiten adaptarse al sistema universitario y responder a las exigencias académicas. En el nivel meso se incluyen las relaciones con la familia, los docentes y el grupo de pares y que pueden tener influencia en el desarrollo académico. Finalmente, a nivel macro se evalúan los aspectos institucionales que incluyen las condiciones económicas, las políticas educativas, empleabilidad y creencias religiosas.

En el microsistema se encuentran las escalas: a) Intrapersonal (percepción del docente sobre la capacidad de comprensión, resolución de problemas, interés y decisión vocacional que realiza el estudiante para responder a las demandas académicas dentro del contexto universitario); y b) adaptación personal (percepción del docente de los ajustes a nivel cognitivo y conductual que realiza el estudiante para afrontar las demandas y exigencias académicas de la universidad).

Por su parte, en el mesosistema universitario se evalúan dos escalas: a) redes de apoyo disfuncional (amenazas percibidas por el profesor acerca de las relaciones con familiares y pares, que no brindan soporte para el desarrollo de las actividades académicas y afectan la permanencia en la Universidad); b). Interacción docente-estudiante (formas de comunicación y relación que emergen en las relaciones pedagógicas, procesos de aprendizaje e interacciones con el docente para la permanencia universitaria). Finalmente, en el macrosistema se ubica la escala: a) aspectos institucionales y empleabilidad laboral (percepción que tiene el docente del desconocimiento del estudiante de las políticas, normas institucionales, incentivos y bajas expectativas laborales del programa cursado). 
Tabla 1.

Distribución de percentiles CDUp

\begin{tabular}{|c|c|c|c|c|c|c|c|c|c|c|c|c|c|c|}
\hline \multicolumn{6}{|c|}{ NIVEL MICRO } & \multicolumn{6}{|c|}{ NIVEL MESO } & \multirow{2}{*}{\multicolumn{3}{|c|}{$\begin{array}{l}\text { NIVEL MACRO } \\
\text { ASPECTOS INSI- } \\
\text { TUCIONALES Y } \\
\text { EMPLEABILIDAD }\end{array}$}} \\
\hline \multicolumn{3}{|c|}{ INTRAPERSONAL } & \multicolumn{3}{|c|}{ ADAPTACIÓN } & \multicolumn{3}{|c|}{ RED DE APOYO SOCIAL } & \multicolumn{3}{|c|}{$\begin{array}{c}\text { INTERACCIÓN } \\
\text { DOCENTE-ESTUDIANTE }\end{array}$} & & & \\
\hline $\begin{array}{l}\text { Sin } \\
\text { riesgo }\end{array}$ & $\begin{array}{c}\text { Mediano } \\
\text { riesgo }\end{array}$ & $\begin{array}{l}\text { Alto } \\
\text { riesgo }\end{array}$ & $\begin{array}{l}\text { Sin } \\
\text { riesgo }\end{array}$ & $\begin{array}{l}\text { Mediano } \\
\text { riesgo }\end{array}$ & $\begin{array}{l}\text { Alto } \\
\text { riesgo }\end{array}$ & $\begin{array}{l}\text { Sin } \\
\text { riesgo }\end{array}$ & $\begin{array}{c}\text { Mediano } \\
\text { riesgo }\end{array}$ & $\begin{array}{l}\text { Alto } \\
\text { riesgo }\end{array}$ & $\begin{array}{l}\text { Sin } \\
\text { riesgo }\end{array}$ & $\begin{array}{c}\text { Mediano } \\
\text { riesgo }\end{array}$ & $\begin{array}{l}\text { Alto } \\
\text { riesgo }\end{array}$ & $\begin{array}{l}\text { Sin } \\
\text { riesgo }\end{array}$ & $\begin{array}{c}\text { Mediano } \\
\text { riesgo }\end{array}$ & $\begin{array}{l}\text { Alto } \\
\text { riesgo }\end{array}$ \\
\hline $0-20$ & $21-27$ & $28-40$ & $0-22$ & $23-28$ & $29-48$ & $0-26$ & 26- 34 & $35-52$ & $0-18$ & $19-26$ & $27-44$ & $0-14$ & $15-22$ & $23-48$ \\
\hline
\end{tabular}

\subsection{Diseño y procedimiento}

Estudio instrumental (Montero \& León, 2007) en el que se analizó las evidencias de validez de contenido y de constructo del CDUp. La validez de contenido fue examinada a partir del acuerdo interjueces, realizada por nueve expertos (en psicología, en psicometría y en deserción universitaria) (Kappa $\mathrm{CCl}=0,85$ ). Producto del análisis se realizaron ajustes de redacción y se eliminaron ítems que no resultaron claros ni representativos del constructo. Esta investigación contó con el aval del Comité de ética de la Universidad Pedagógica y Tecnológica de Colombia; en su ejecución, se salvaguardaron todos los principios éticos y normas legales que rigen la investigación en Salud y en Psicología. Previa autorización de las dos instituciones educativas superiores, se tuvo acceso a la base de datos de los profesores de ambas universidades, luego se procedió a seleccionar y contactar a los participantes, a quienes se les informó sobre el propósito de la investigación, el nivel de riesgo, los beneficios, el carácter voluntario de la participación y la confidencialidad de la información. Por último, los participantes firmaron el consentimiento informado y diligenciaron el instrumento. Las aplicaciones se realizaron de mayo a diciembre de 2019.

\subsection{Plan de análisis}

El análisis de los datos se realizó con el programa Factor 10.3.01. Se examinaron los supuestos de distribución normal a partir de los valores de Kolmogorov Smirnov y la homocedasticidad de la varianza mediante la prueba de Levene para verificar el uso de pruebas paramétricas. Posteriormente, se evaluó la adecuación del análisis factorial mediante la prueba de esfericidad de Bartlett y el KMO. Para el análisis factorial exploratorio se utilizó el procedimiento Parallel Análisis a fin de determinar qué estructura factorial explica mejor la variabilidad de los ítems, asimismo, se usó el método de mínimos cuadrados, rotación promax y matriz de correlaciones policóricas. Para caracterizar la muestra se identificó la media y la desviación estándar de acuerdo al género (hombre-mujer), y el tipo de universidad (pública-privada) 
para cada escala de CDUp. También, se halló la distribución en percentiles estableciendo tres niveles de valoración de qué tanto riesgo de deserción representa para el docente cada uno de los cinco factores. El análisis de la relación entre variables se realizó mediante el coeficiente de correlación de Pearson. Finalmente, el análisis de la fiabilidad se realizó mediante el método Alpha de Cronbach basado en la varianza del test y el método de fiabilidad compuesta Omega de McDonalds basado en la carga factorial de cada ítem; este último realizado con el programa JASP.

\section{RESULTADOS}

\subsection{Análisis descriptivo e inferencial}

El análisis descriptivo arrojó que los profesores de la universidad pública y privada perciben mayor riesgo en la escala Red de apoyo disfuncional, seguida de la escala Intrapersonal notándose mayores puntuaciones en los profesores de la universidad pública (Tabla 2).

Tabla 2.

Análisis descriptivos del CDUp

\begin{tabular}{|c|c|c|c|c|c|c|c|c|c|c|c|c|c|c|c|c|}
\hline \multirow{3}{*}{ GÉNERO } & \multirow[t]{3}{*}{$\begin{array}{c}\text { TIPO DE } \\
\text { UNIVERSIDAD }\end{array}$} & & & & \multirow{2}{*}{\multicolumn{3}{|c|}{$\begin{array}{l}\text { NIVEL MICRO } \\
\text { ADAPTACIÓN } \\
\text { PERSONAL }\end{array}$}} & \multicolumn{6}{|c|}{ NIVEL MESO } & \multirow{2}{*}{\multicolumn{3}{|c|}{$\begin{array}{c}\text { NIVEL MACRO } \\
\text { ASPECTOS } \\
\text { INSTITUCIONALES }\end{array}$}} \\
\hline & & \multicolumn{3}{|c|}{ INTRAPERSONAL } & & & & \multicolumn{3}{|c|}{$\begin{array}{l}\text { RED DE APOYO } \\
\text { DISFUNCIONAL }\end{array}$} & \multicolumn{3}{|c|}{$\begin{array}{l}\text { INTERACCIÓN } \\
\text { DOCENTE- } \\
\text { ESTUDIANTE }\end{array}$} & & & \\
\hline & & $\mathbf{N}$ & M & DE & $\mathbf{N}$ & M & $\mathrm{DE}$ & $\mathbf{N}$ & M & $\mathrm{DE}$ & $\mathbf{N}$ & $\mathbf{M}$ & $\mathrm{DE}$ & $\mathbf{N}$ & M & DE \\
\hline \multirow[b]{2}{*}{ Femenino } & Publica & 201 & 2,23 & 0,63 & 201 & 2,10 & 0,74 & 201 & 2,32 & 0,75 & 201 & 2,06 & 0,87 & 201 & 1,69 & 0,78 \\
\hline & Privada & 143 & 2,21 & 0,69 & 143 & 2,17 & 0,77 & 143 & 2,28 & 0,82 & 143 & 2,05 & 1,00 & 143 & 1,60 & 0,88 \\
\hline \multirow{2}{*}{ Masculino } & Publica & 52 & 2,28 & 0,61 & 52 & 2,08 & 0,59 & 52 & 2,22 & 0,75 & 52 & 2,04 & 0,77 & 52 & 1,60 & 0,78 \\
\hline & Privada & 68 & 2,01 & 0,56 & 68 & 2,03 & 0,68 & 68 & 2,24 & 0,71 & 68 & 1,73 & 0,82 & 68 & 1,41 & 0,70 \\
\hline
\end{tabular}

El análisis ANOVA de una vía al comparar años de experiencia docente con respecto a las puntuaciones promedio de cada una de las escalas, arrojó diferencias estadísticamente significativas para las escalas Intrapersonal ( $F$ $(g \mathrm{l}=2, n=464)=4,190, p=0,016)$ y Adaptación Personal $\left(F_{(g l=2, n=464)}=3,051\right.$, $p=0,04)$. En ambas escalas, los profesores que tienen más de 15 años de experiencia otorgan mayor nivel de riesgo. El análisis $t$ student de acuerdo al género, reportó diferencias significativas en la escala Interacción estudiante-docente, siendo las profesoras quienes otorgan mayores puntuaciones en esta escala como indicador de riesgo de deserción de los estudiantes $\left(\mathrm{t} \mathrm{gl}_{\mathrm{g}}=\right.$ $724, n=708)=2,084, p=0,038$ ). Por su parte, el análisis t student para muestras independientes no arrojó diferencias estadísticamente significativas entre el 
tipo de universidad y las puntuaciones promedio de las diferentes escalas, ni entre las facultades con altas y bajas tasas de deserción ( $p>0,05)$.

Para finalizar, el análisis intersujetos en la prueba multivariante $(3 \times 2 \times 2)$ indicó que, existe una interacción estadísticamente significativa entre el tipo de universidad (pública y privada) y el género (mujer u hombre) para las puntuaciones promedio de la escala Adaptación Personal $\left(F_{(\mathrm{gl}=1, n=464)}=\right.$ $\left.6,453, p=0,011 ; \eta^{2}=0,021\right)$; el riesgo percibido de deserción en esta escala fue mayor para las profesoras de la universidad privada. Adicionalmente, para esta misma escala, el análisis arrojó una interacción estadísticamente significativa entre universidad, años de experiencia y género $\left(F_{(g l=2, n=464)}=\right.$ $\left.3,338, p=0,036 ; \eta^{2}=0,021\right)$, indicando que las profesoras de la universidad privada y con mayor experiencia perciben mayor riesgo de deserción en los estudiantes desde esta escala.

\subsection{Estructura Interna}

A partir de la adecuación del análisis factorial (KMO $=0,951$; Bartlett's test $\left.\chi_{(g l=4095 ; n=464)}^{2}=19465,0, p=0,000\right)$ se procedió a realizar un Análisis Factorial Exploratorio (AFE) mediante el procedimiento de extracción de Parallel Análisis y el método de extracción de mínimos cuadrados y rotación promax. Para la extracción de ítems se guardó el criterio de carga factorial por ítem mínima de 0,40. La Tabla 3 muestra el resultado del análisis factorial exploratorio.

Tabla 3.

Análisis factorial exploratorio del CDUp

\begin{tabular}{|c|c|c|c|c|c|}
\hline \multirow[t]{2}{*}{ FACTORES } & \multicolumn{2}{|c|}{ COMPONENTES } & & \multicolumn{2}{|c|}{ VARIANZA EXPLICADA } \\
\hline & NIVEL & ESCALAS & ÍTEMS & $\%$ VARIANZA & $\%$ ACUMULADO \\
\hline 1 & Macro & $\begin{array}{l}\text { Aspectos instituciona- } \\
\text { les y empleabilidad }\end{array}$ & $\begin{array}{c}76,78,79,80,82,83 \\
84,86,87,88,89\end{array}$ & 35,75 & 35,75 \\
\hline 2 & Meso & $\begin{array}{l}\text { Redes de apoyo } \\
\text { disfuncional }\end{array}$ & $\begin{array}{c}30,32,55,56,57,61 \\
64,66,67,70,71 \\
72,73\end{array}$ & 5,23 & 43,6 \\
\hline 3 & Micro & Intrapersonal & $\begin{array}{c}2,3,4,6,7,8,9,10 \\
12,14,19\end{array}$ & 3,15 & 48,45 \\
\hline 4 & Meso & $\begin{array}{c}\text { Interacción } \\
\text { estudiante-docente }\end{array}$ & $\begin{array}{c}41,42,43,44,45,46 \\
47,49,50,51,54\end{array}$ & 2,43 & 52,15 \\
\hline 5 & Micro & Adaptación Personal & $\begin{array}{c}20,21,22,23,24 \\
28,35,36,37,38 \\
39,40\end{array}$ & 2,06 & 55,28 \\
\hline
\end{tabular}


El AFE para el CDUp agrupó 64 ítems en total, de los cuales se eliminaron 6 ítems que no guardaron correspondencia teórica con los ítems agrupados en la matriz de la estructura del análisis factorial. La Tabla 4 muestra el resultado de los cinco componentes conformados con los 59 ítems seleccionados.

Tabla 4.

Matriz de ítems seleccionados para CDUp

\begin{tabular}{|c|c|c|c|c|c|c|c|}
\hline COMPONENTE & & ÍTEM & M & $\mathrm{DE}$ & G1 & G2 & $\begin{array}{l}\text { CARGA } \\
\text { FACTORIAL }\end{array}$ \\
\hline \multirow{11}{*}{$\begin{array}{l}\text { Micro: } \\
\text { Intrapersonal }\end{array}$} & 2 & Presenta dificultades para resolver las tareas académicas. & 2,45 & 0,983 & $-0,324$ & $-0,27$ & 0,709 \\
\hline & 3 & Presenta dificultades para seguir instrucciones. & 2,29 & 0,984 & $-0,224$ & $-0,40$ & 0,616 \\
\hline & 4 & $\begin{array}{c}\text { Utiliza estrategias de estudio que son poco efectivas en su } \\
\text { proceso de aprendizaje. }\end{array}$ & 2,44 & 1,040 & $-0,371$ & $-0,31$ & 0,734 \\
\hline & 6 & $\begin{array}{c}\text { Se percibe poco habilidoso para desarrollar las tareas } \\
\text { académicas. }\end{array}$ & 2,21 & 0,964 & $-0,181$ & $-0,179$ & 0,695 \\
\hline & 7 & $\begin{array}{l}\text { Obtiene bajas calificaciones en los parciales, quices, talleres, } \\
\qquad \text { o controles de lectura. }\end{array}$ & 2,548 & 1,004 & $-0,251$ & $-0,590$ & 0,699 \\
\hline & 8 & No prepara el material de lectura para la clase. & 2,363 & 1,082 & $-0,249$ & $-0,573$ & 0,798 \\
\hline & 9 & Carece de estrategias, métodos y hábitos de estudio. & 2,690 & 0,997 & $-0,452$ & $-0,430$ & 0,825 \\
\hline & 10 & $\begin{array}{l}\text { Se le dificulta resolver los problemas que se le plantean en } \\
\text { clase }\end{array}$ & 2,44 & 0,955 & $-0,284$ & $-0,197$ & 0,710 \\
\hline & 12 & No identificarse con el plan de estudios que cursa. & 2,514 & 1,210 & $-0,439$ & $-0,749$ & 0,469 \\
\hline & 14 & $\begin{array}{l}\text { Presenta baja motivación en el desarrollo de las actividades } \\
\text { de clase. }\end{array}$ & 2,428 & 1,016 & $-0,302$ & $-0,358$ & 0,476 \\
\hline & 19 & $\begin{array}{l}\text { Percibe que las asignaturas no son relevantes para su forma- } \\
\text { ción profesional. }\end{array}$ & 2,325 & 1,108 & $-0,306$ & $-0,548$ & 0,412 \\
\hline \multirow{12}{*}{$\begin{array}{l}\text { Micro: Adapta- } \\
\text { ción Personal }\end{array}$} & 20 & Incumple con la entrega de trabajos. & 2,733 & 1,015 & $-0,603$ & $-0,080$ & 0,471 \\
\hline & 21 & Tiene baja participación en clase. & 2,108 & 1,121 & $-0,065$ & $-0,636$ & 0,688 \\
\hline & 22 & Llega tarde a la mayoría de las clases. & 2,140 & 1,140 & $-0,066$ & $-0,754$ & 0,750 \\
\hline & 23 & No asiste a las tutorías programadas. & 2,191 & 1,197 & $-0,163$ & $-0,881$ & 0,755 \\
\hline & 24 & No usa con regularidad el aula virtual. & 1,544 & 1,152 & 0,309 & $-0,782$ & 0,699 \\
\hline & 28 & $\begin{array}{c}\text { Se frustra cuando no comprende los contenidos de la } \\
\text { asignatura. }\end{array}$ & 2,247 & 0,985 & $-0,114$ & $-0,408$ & 0,435 \\
\hline & 35 & $\begin{array}{l}\text { Presenta frecuentemente excusas para evadir sus responsabi- } \\
\text { lidades académicas. }\end{array}$ & 2,544 & 1,023 & $-0,311$ & $-0,530$ & 0,491 \\
\hline & 36 & $\begin{array}{l}\text { Se Frustra cuando no consigue tener buenas calificaciones en } \\
\qquad \text { la asignatura. }\end{array}$ & 2,110 & 0,949 & $-0,169$ & $-0,336$ & 0,529 \\
\hline & 37 & $\begin{array}{l}\text { Presenta problemas para adaptarse a las dinámicas de la } \\
\text { universidad. }\end{array}$ & 2,082 & 1,000 & $-0,148$ & $-0,370$ & 0,513 \\
\hline & 38 & Tiene problemas para adaptarse a la metodología de clase. & 2,092 & 1,032 & $-0,065$ & $-0,584$ & 0,455 \\
\hline & 39 & $\begin{array}{c}\text { No identificarse con las dinámicas y funcionamiento de la } \\
\text { institución. }\end{array}$ & 1,763 & 1,091 & 0,071 & $-0,756$ & 0,474 \\
\hline & 40 & $\begin{array}{c}\text { Recibe con frecuencia llamados de atención de los } \\
\text { profesores. }\end{array}$ & 1,772 & 1,077 & 0,080 & $-0,670$ & 0,415 \\
\hline
\end{tabular}




\begin{tabular}{|c|c|c|c|c|c|c|c|}
\hline COMPONENTE & & ÍTEM & M & DE & G1 & G2 & $\begin{array}{l}\text { CARGA } \\
\text { FACTORIAL }\end{array}$ \\
\hline \multirow{11}{*}{$\begin{array}{c}\text { Meso: } \\
\text { Interacción estu- } \\
\text { diante-docente }\end{array}$} & 41 & $\begin{array}{c}\text { Se siente insatisfecho con el nivel de formación de sus } \\
\text { docentes. }\end{array}$ & 1,735 & 1,229 & 0,213 & $-0,872$ & 0,691 \\
\hline & 42 & $\begin{array}{l}\text { Percibe que las estrategias pedagógicas que utiliza el docente } \\
\text { no facilitan la comprensión de los contenidos. }\end{array}$ & 1,944 & 1,133 & 0,012 & $-0,686$ & 0,578 \\
\hline & 43 & $\begin{array}{l}\text { Usar un modelo de educación tradicional le dificulta al estu- } \\
\text { diante su aprendizaje. }\end{array}$ & 1,768 & 1,092 & 0,103 & $-0,656$ & 0,671 \\
\hline & 44 & $\begin{array}{l}\text { Percibe que no existe relación entre lo que el docente enseña } \\
\qquad y \text { lo que el docente evalúa. }\end{array}$ & 2,06 & 1,114 & $-0,131$ & $-0,647$ & 0,880 \\
\hline & 45 & $\begin{array}{l}\text { No existe relación entre la teoría y la práctica en el desarrollo } \\
\text { de la asignatura. }\end{array}$ & 2,082 & 1,176 & $-0,152$ & $-0,822$ & 0,987 \\
\hline & 46 & Percibe que no hay un hilo conductor en las clases. & 1,991 & 1,163 & $-0,157$ & $-0,757$ & 0,939 \\
\hline & 47 & $\begin{array}{l}\text { Percibe que los métodos de evaluación que emplea el docen- } \\
\text { te no le permiten dar cuenta de sus habilidades. }\end{array}$ & 1,974 & 1,146 & $-0,062$ & $-0,827$ & 0,895 \\
\hline & 49 & Ha sido víctima de maltrato por parte de sus profesores. & 2,277 & 1,321 & $-0,367$ & $-1,011$ & 0,485 \\
\hline & 50 & $\begin{array}{l}\text { La exigencia de la asignatura supera las horas de dedicación } \\
\text { que tiene que cumplir el estudiante. }\end{array}$ & 2,080 & 1,170 & $-0,156$ & $-0,796$ & 0,534 \\
\hline & 51 & $\begin{array}{l}\text { No recibe retroalimentación a tiempo por parte del docente, } \\
\text { para generar acciones de cambio. }\end{array}$ & 2,045 & 1,142 & $-0,211$ & $-0,726$ & 0,598 \\
\hline & 54 & Recibe feedback negativo sobre sus capacidades académicas. & 2,088 & 1,077 & $-0,206$ & $-0,561$ & 0,543 \\
\hline \multirow{13}{*}{$\begin{array}{c}\text { Meso: } \\
\text { Red de apoyo } \\
\text { Disfuncional }\end{array}$} & 30 & Percibe poco apoyo familiar en su vida académica. & 2,510 & 1,084 & $-0,499$ & $-0,344$ & 0,578 \\
\hline & 32 & Se aísla de su grupo de compañeros. & 2,288 & 1,140 & $-0,119$ & $-0,826$ & 0,446 \\
\hline & 55 & $\begin{array}{l}\text { Atravesar por la enfermedad de un familiar agota emocional- } \\
\text { mente al estudiante. }\end{array}$ & 2,624 & 1,021 & $-0,384$ & $-0,429$ & 0,943 \\
\hline & 56 & $\begin{array}{c}\text { Ser víctima de maltrato intrafamiliar (físico, psicológico) } \\
\text { afecta su desempeño académico. }\end{array}$ & 2,867 & 1,073 & $-0,787$ & 0,000 & 1,032 \\
\hline & 57 & $\begin{array}{c}\text { Cuidar de algún familiar le impide el cumplimiento de los } \\
\text { trabajos académicos. }\end{array}$ & 2,682 & 1,061 & $-0,569$ & $-0,272$ & 0,900 \\
\hline & 61 & $\begin{array}{c}\text { Los padres no aprueban la elección de carrera que realizó el } \\
\text { estudiante. }\end{array}$ & 1,858 & 1,200 & 0,109 & $-0,885$ & 0,452 \\
\hline & 64 & $\begin{array}{l}\text { Los padres de familia no se involucran ni apoyan el proceso } \\
\text { educativo de los hijos. }\end{array}$ & 1,796 & 1,196 & 0,164 & $-0,866$ & 0,423 \\
\hline & 66 & Le cuesta integrarse con sus compañeros de clase. & 1,82 & 1,063 & 0,017 & $-0,552$ & 0,530 \\
\hline & 67 & $\begin{array}{c}\text { Es víctima de maltrato (psicológico, físico, verbal) por parte } \\
\text { de sus compañeros. }\end{array}$ & 2,484 & 1,208 & $-0,521$ & $-0,621$ & 0,738 \\
\hline & 70 & $\begin{array}{c}\text { No cuenta con un grupo de amigos en los que confíe y } \\
\text { busque apoyo. }\end{array}$ & 1,938 & 1,091 & $-0,014$ & $-0,657$ & 0,665 \\
\hline & 71 & $\begin{array}{l}\text { Los compañeros lo excluyen de los trabajos y actividades } \\
\text { académicas. }\end{array}$ & 2,140 & 1,111 & $-0,164$ & $-0,706$ & 0,750 \\
\hline & 72 & $\begin{array}{l}\text { Tiene un grupo de amigos que prioriza otras actividades que } \\
\text { le impide el cumplimiento de las actividades académicas. }\end{array}$ & 1,994 & 1,065 & $-0,114$ & $-0,603$ & 0,549 \\
\hline & 73 & $\begin{array}{l}\text { Dedica bastante tiempo a realizar actividades de ocio que le } \\
\text { impiden cumplir con sus obligaciones académicas. }\end{array}$ & 2,703 & 1,080 & $-0,570$ & $-0,423$ & 0,669 \\
\hline
\end{tabular}




\begin{tabular}{|c|c|c|c|c|c|c|c|}
\hline COMPONENTE & & ÍTEM & $M$ & DE & G1 & G2 & $\begin{array}{l}\text { CARGA } \\
\text { FACTORIAL }\end{array}$ \\
\hline \multirow{12}{*}{$\begin{array}{c}\text { Macro: Aspectos } \\
\text { institucionales y } \\
\text { empleabilidad }\end{array}$} & 76 & $\begin{array}{c}\text { Desconoce la modalidad de trabajo de grado que va a utilizar } \\
\text { para graduarse. }\end{array}$ & 1,310 & 1,183 & 0,524 & $-0,709$ & 0,537 \\
\hline & 78 & $\begin{array}{c}\text { Desconoce cuáles son las situaciones por las que puede } \\
\text { perder la calidad de estudiante universitario. }\end{array}$ & 1,903 & 1,260 & 0,039 & $-1,015$ & 0,669 \\
\hline & 79 & $\begin{array}{l}\text { Desconoce los incentivos (i.e. becas, pasantías, movilidad, } \\
\text { etc.) que ofrece la universidad. }\end{array}$ & 1,684 & 1,217 & 0,241 & $-0,867$ & 0,862 \\
\hline & 80 & $\begin{array}{l}\text { No cuenta con una beca que apoye el sostenimiento econó- } \\
\text { mico del estudiante. }\end{array}$ & 1,759 & 1,190 & 0,104 & $-0,933$ & 0,615 \\
\hline & 82 & Desconoce el proyecto educativo de la Universidad. & 1,366 & 1,111 & 0,476 & $-0,522$ & 0,836 \\
\hline & 83 & Desconoce las áreas de aplicación de su carrera. & 1,766 & 1,159 & 0,107 & $-0,854$ & 0,689 \\
\hline & 84 & $\begin{array}{l}\text { La Universidad no ofrece servicios de orientación profesional } \\
\qquad \text { a sus estudiantes. }\end{array}$ & 1,546 & 1,187 & 0,360 & $-0,761$ & 0,590 \\
\hline & 85 & $\begin{array}{l}\text { Otorga mayor importancia a recibir remuneración económica } \\
\text { que a terminar sus estudios. }\end{array}$ & 2,20 & 1,189 & $-0,219$ & $-0,827$ & 0,428 \\
\hline & 86 & Desconoce el reglamento de la institución. & 1,568 & 1,195 & 0,364 & $-0,782$ & 0,754 \\
\hline & 87 & Percibe que su profesión es poco valorada en la comunidad. & 1,660 & 1,173 & 0,122 & $-0,888$ & 0,574 \\
\hline & 88 & $\begin{array}{l}\text { Las creencias religiosas del estudiante le impiden desarrollar } \\
\text { actividades propias de su profesión. }\end{array}$ & 0,959 & 1,087 & 0,918 & $-0,102$ & 0,490 \\
\hline & 89 & Percibe bajos índices de empleo en la ciudad y en el país. & 1,665 & 1,232 & 0,235 & $-0,923$ & 0,665 \\
\hline
\end{tabular}

Nota. $\mathrm{M}=$ Media; $\mathrm{DE}$ = Desviación estándar; g1 = Asimetría; $\mathrm{g} 2$ = Curtosis.

El AFE para el CDUp agrupó 64 ítems en total, de los cuales se eliminaron seis ítems que no guardaron correspondencia teórica con los ítems agrupados en la matriz de la estructura del análisis factorial. La Tabla 4 muestra el resultado de los cinco componentes conformados con los 59 ítems seleccionados.

El análisis de Pearson mostró relación positiva entre las diferentes escalas que componen el instrumento CUDp-3. Las correlaciones se detallan en la Tabla 5.

Tabla 5.

Correlaciones de Pearson para las escalas del CDUp

\begin{tabular}{cccccc}
\hline NIVELES & ESCALAS & (A) & (B) & (C) & (D) \\
\hline Micro & Intrapersonal (A) & & & \\
& Adaptación Personal (B) & $0,668^{* *}$ & & \\
Meso & Red de Apoyo social (C) & $0,417^{* *}$ & $0,536^{* *}$ & & \\
& Interacción estudiante-docente (D) & $0,525^{* *}$ & $0,568^{* *}$ & $0,683^{* *}$ & \\
& Aspectos institucionales y emplea- & $0,455^{* *}$ & $0,543^{* *}$ & $0,611^{* *}$ & $0,549^{* *}$ \\
Milidad (F) & & & &
\end{tabular}




\subsection{Consistencia interna}

Determinada a partir del análisis de fiabilidad mediante alfa de Cronbach y McDonald, se concluyó que, los ítems agrupados en cada componente están relacionados recíprocamente en CDUp. El Alpha de Cronbach reveló niveles de fiabilidad fuerte $(\alpha=0,89-0,91)$ en las cinco escalas y en la prueba total. La fiabilidad compuesta de McDonalds reveló niveles de fiabilidad fuerte ( $\omega$ $=0,89-0,93$ ) para todas las escalas (ver Tabla 6).

Tabla 6.

Análisis de fiabilidad CDUp

\begin{tabular}{cccccc}
\hline NIVEL & ESCALA & TOTAL ÍtEMS & $\begin{array}{c}\text { OMEGA DE } \\
\text { MCDONALD ( } \Omega \text { ) }\end{array}$ & $\begin{array}{c}\text { ALPHA DE } \\
\text { CRONBACH (A) }\end{array}$ & $\begin{array}{c}\text { INTERVALO DE } \\
\text { CONFIANZA 95 \% }\end{array}$ \\
\hline \multirow{2}{*}{ Micro } & Intrapersonal & 11 & 0,9 & 0,89 & $0,88-0,89$ \\
& $\begin{array}{c}\text { Adaptación Personal } \\
\text { Redes de apoyo }\end{array}$ & 12 & 0,89 & 0,89 & $0,88-0,90$ \\
Meso & $\begin{array}{c}\text { disfuncional } \\
\text { Interacción }\end{array}$ & 13 & 0,91 & 0,91 & $0,89-0,92$ \\
& $\begin{array}{c}\text { estudiante-docente } \\
\text { Macro }\end{array}$ & 11 & 0,93 & 0,93 & $0,92-0,94$ \\
& $\begin{array}{c}\text { Aspectos } \\
\text { institucionales }\end{array}$ & 12 & 0,89 & 0,89 & $0,88-0,90$ \\
& Total CDUp & 59 & 0,95 & 0,95 & $0,95-0,96$ \\
\hline
\end{tabular}

\section{DISCUSIÓN}

Los resultados del estudio muestran la validez y la confiabilidad del CDUp para evaluar la percepción de los docentes sobre los factores de riesgo de deserción en estudiantes universitarios. El cuestionario responde en su diseño y validación al modelo y revisión teórica realizada para su construcción (i.e. modelo ecológico y deserción escolar), teniendo en cuenta que los datos se agruparon de acuerdo a los tres entornos ecológicos: micro, meso y macro. EI CDUp, es una herramienta objetiva para identificar la percepción de los docentes sobre el fenómeno de la deserción. Adicionalmente, la información arrojada a partir del cuestionario se convierte en un insumo clave para el diseño de programas de intervención con docentes, en los que se fortalezca una percepción ecológica de la deserción, reconociendo como relevantes todos los sistemas de interacción del estudiante. Los análisis muestran una correlación significativa entre las diferentes escalas que componen el instrumento CUDp aportando evidencia en la relación que existe en los distintos sistemas de interacción del sujeto.

El análisis de fiabilidad evidenció que los ítems que hacen parte de cada componente se encuentran relacionados entre sí. Así mismo, el AFE, arrojó una estructura pentafactorial en la que se agruparon 59 ítems en las 
escalas de Intrapersonal y Adaptación personal -nivel micro-; las escalas de interacción estudiante-docente y Redes de apoyo disfuncional -nivel mesoy Aspectos institucionales y empleabilidad -nivel macro-. La conformación de cada escala, guardó relación con la evidencia empírica revisada sobre las variables que son relevantes en cada uno de los subsistemas de interacción del estudiante y sobre el papel que tiene el docente en la decisión que toma un estudiante de desertar (Gil et al., 2018).

En el estudio se encontró que los profesores de más experiencia en procesos de enseñanza, dan mayor importancia a las escalas Intrapersonal y Adaptación Personal, lo que implica que, ellos reconocen como relevantes los factores de tipo individual (i.e., capacidad de comprensión, resolución de problemas, interés y decisión vocacional) y la capacidad del estudiante para adaptarse al sistema universitario y responder a las exigencias académicas (Gil et al., 2018). Este resultado se relaciona con el estudio realizado por Tejedor y García-Valcárcel (2007) donde los profesores de mayor antigüedad dan mayor relevancia al bajo rendimiento académico que afecta los procesos de adaptación por el exceso de trabajo y la exigencia del profesorado, además, de no cursar la carrera elegida como primera opción, que estaría relacionado con la elección vocacional.

Los hallazgos de este estudio aportan evidencia para considerar que el fenómeno de la deserción, es de naturaleza multicausal y multidimensional donde interactúan una multiplicidad de variables y actores que configuran los distintos subsistemas del contexto universitario: micro, meso y macro. El instrumento CDUp se constituye en una herramienta valiosa para aportar en la evaluación de este fenómeno considerando los aspectos principales en cada uno de estos sistemas. A nivel micro, desde la capacidad de adaptación personal y las habilidades cognitivas, motivacionales y vocacionales; el nivel meso desde las redes de apoyo (i.e., familia y grupo de pares) e interacción estudiante-docente y el nivel macro estructural aspectos institucionales y empleabilidad.

En esta línea, se sugiere abordar la deserción universitaria a partir, de modelos que permitan explicar, de forma suficiente, este fenómeno teniendo en cuenta tanto la percepción del estudiante como la del docente, para captar desde los múltiples actores los factores asociados a la deserción considerando que el docente es quien tiene contacto directo con el estudiante y es quien puede detectar de forma oportuna algún riesgo de deserción. Asimismo, desde sus acciones en el aula, el profesor puede ayudar al estudiante a gestionar distintos estados emocionales, aumentar la motivación y nivel de 
compromiso, lo que redundará en una mayor posibilidad de permanecer en el sistema universitario (Ottosen et al., 2016; Quin, 2016; Rumberger \& Rotermund, 2012).

Finalmente, se sugiere usar el CDUp para realizar estudios que articulen diferentes variables de personalidad, funcionamiento cognitivo, socioculturales, adaptación, redes de apoyo, desempeño académico, entre otras, que permita articular la percepción de otros actores del sistema, a través, de modelos estadísticos robustos, por ejemplo, análisis de ecuaciones estructurales o análisis jerarquizados, que permitan explicar de forma suficiente el fenómeno de la deserción. En este sentido, el cuestionario puede ser usado dentro de las baterías de tamizaje que permitan evaluar el riesgo de forma global. De igual forma, se sugiere utilizar el instrumento para orientar programas de intervención ajustados a la evidencia empírica reportada, teniendo en cuenta los tipos de interacción entre las distintas variables.

\section{CONCLUSIÓN}

El CDUp es un instrumento basado en el modelo bioecológico de Bronfrenbrenner, que muestra adecuados niveles de confiablidad y validez para evaluar la percepción de los docentes sobre los factores de riesgos de deserción universitaria. Este instrumento, junto a otras herramientas de detección como autorreportes y/o entrevistas con estudiantes, permitirán a las IES orientar políticas institucionales y planes de acción para disminuir las tasas de deserción. Adicionalmente, el CDUp es una herramienta objetiva sobre la valoración que hace el profesor de la deserción que permitirá a las IES ampliar las comprensiones que existen alrededor de este fenómeno, considerando no solo su naturaleza multicausal y multidimensional, sino que parte de un abordaje del desarrollo y ajuste del estudiante al sistema universitario desde los procesos de interacción que establece en los distintos sistemas próximos (e.g, familia, docente, pares) y distales (e.g., políticas educativas, contexto laboral, cultura).

Agradecimiento: A lo docentes universitarios de las dos instituciones de educación superior que amablemente participaron en el estudio. Al grupo de investigación Desarrollo Humano, cognición y Educación, por sus aportes en la recolección de información.

Conflicto de interés: El artículo no presenta un conflicto potencial de interés, los resultados que se muestran se derivan del proyecto de investigación Modelo Psicosocial de alertas tempranas de deserción universitaria académica y no académica, código SGI 2395. 
Financiamiento: Este estudio hace parte del Proyecto Modelo Psicosocial de alertas tempranas de deserción universitaria académica y no académica, código SGI 2395, financiado por la Universidad pedagógica y Tecnológica de Colombia y la Universidad de Boyacá.

\section{REFERENCIAS}

Aparicio, M. (2012). La deserción universitaria como Parámetro de calidad y su relación con Factores psicosociales. Diálogo Canoas, 20, 145-166. https:// revistas.unilasalle.edu.br/index.php/Dialogo/article/view/284

Astin, A. (1985). Achieving educational excellence: A Critical Assessment of Priorities and Practices in Higher Education (1a ed.). Jossey-Bass.

Bal, S., Mousavi, S. M., Masalimova, A., Bersanov, A., Kurbanov, R., Boiarchuk, A., \& Pavlushin, A. (2018). The Impacts of Teacher's Efficacy and Motivation on Student's Academic Achievement in Science Education among Secondary and High School Students. Eurasia Journal of Mathematics, Science and Technology Education, 14(6), 2353-2366. http://10.29333/ejmste/89579

Barragán, D. \& Patiño, L. (2013). Elementos para la comprensión del fenómeno de la deserción universitaria en Colombia. Más allá de las mediciones. Cuadernos Latinoamericanos de Administración, IX(16), 55-66. https://doi.org/10.18270/ cuaderlam.v9i16.1248

Bronfenbrenner, U. (1983). Ecological models of human development. In: International Encyclopedia of Education (2.Eds.). New York: Oxford, Elsevier (pp.37- 43).

Bronfenbrenner, U. (1995). Developmental ecology through space and time: A future perspective. In P. Moen, G. H. Elder, Jr., \& K. Lüscher (Eds.), Examining lives in context: Perspectives on the ecology of human development (p.619-647): American Psychological Association.

Bronfenbrenner, U. (1987). La ecología del desarrollo humano: experimentos en entornos naturales y diseñados. Paidós.

Bronfenbrenner, U. (2011). Bioecologia do desenvolvimento humano: tornando seres humanos mais humanos. Porto Alegre: Artmed.

Cabrera, A. F., Mejías P. P. \& Fernández, L. (2015). Evolución de perspectivas en el estudio de la retención universitaria en los EEUU: bases conceptuales y puntos de inflexión. En P. Figuera (Ed.). Persistir con éxito en la universidad: de la investigación a la acción (pp 15-40). Barcelona: Editorial Laertes. https://www. researchgate.net/publication/261699533_Evolucion_de_perspectivas_en_el_ estudio_de_la_retencion_universitaria_en_los_EEUU_Bases_conceptuales_y_ puntos_de_inflexion

Díaz, K. \& Osuna, L. (2017). La percepción y práctica docente en relación al abandono escolar en centros de educación media superior de Baja California. XVI Congreso Nacional de Investigación Educativa. San Luis de Potosi. http://www.comie.org. $\mathrm{mx} /$ congreso/memoriaelectronica/v14/doc/0708.pdf

Ferreyra, M., Avitabile, C., Botero, J., Haimovich, F. \& Urzúa, S. (2017). Momento decisivo. La educación superior en América Latina y el Caribe. Momento decisivo. Grupo Banco Mundial. https://openknowledge.worldbank.org/bitstream/ handle/10986/26489/211014ovSP.pdf?sequence=5\&isAllowed=y

Gil, A., Antelm-Lanzat, A., Cacheiro-González, M., \& Pérez-Navío, E. (2018). School dropout factors: a teacher and school manager perspective. Educational Studies, 1-14. https://doi.org/10.1080/03055698.2018.1516632

Giovagnoli, P. (2002). Determinantes de la deserción y graduación universitaria: una aplicación utilizando modelos de duración (Tesis de maestría, Universidad 
Nacional de la Plata). Repositorio Institucional de la UNLP. Recuperada de http:// sedici.unlp.edu.ar/handle/10915/3436

González, C., Montenegro, H., López, L., Munita, I. \& Collao, P. (2011). Relación entre la experiencia de Aprendizaje de estudiantes universitarios y la docencia de sus profesores. Calidad en la educación, 35, 21-49. http://dx.doi.org/10.31619/ caledu.n35.95

González-Rodríguez, D., Vieira, M \& Vidal, J. (2019). Factors that influence early school leaving: a comprehensive model. Educational Research, 1-17. https://doi. org/10.1080/00131881.2019.1596034

Martínez-González, R. \& Álvarez-Blanco, L. (2005). Fracaso y abandono escolar Educación Secundaria Obligatoria: implicación de la familia y centros escolares. Aula abierta, 85, 127-146. http://hdl.handle.net/10651/26950

Márquez, D., Ortiz, S., \& Rendón, M. (2008). Cuestionario de Vivencias Académicas en su versión reducida (QVA-r): un análisis psicométrico. Revista Colombia de Psicología, 18(1), 33-52. https://www.redalyc.org/articulo.oa?id=80412413004

Mejía, P., Vélez, L., Martínez, C. Restrepo, S., Zarrabe, C. Lopera, J., Roldán, J. Rincón, A., Marín, L. \& Cárdenas, J. (2016). La tutoría académica. Una alternativa para la superación del rezago académico y la deserción universitaria (1.a ed.). Universidad CES. http://repository.ces.edu.co/bitstream/10946/3786/1/La_tutoria_ academica.pdf

Melo-Becerra, L., Ramos-Forero, J. \& Hernández-Santamaría, P. (2017). La educación superior en Colombia: situación actual y análisis de eficiencia. Revista Desarrollo y Sociedad, 78, 59-111. https://doi.org/10.13043/dys.78.2

Montero, I. \& León, O. (2007). A guide for naming research studies in Psychology. International Journal of Clinical and Health Psychology, 7(3), 847-862. https:// www.researchgate.net/publication/26495811

Moreira-Mora, T. (2007). Perfil sociodemográfico y académico de estudiantes en deserción del sistema educativo. Actualidades en Psicología, 27, 145-165. https:// doi.org/10.15517/ap.v21i108.31

Observatorio de Educación Superior de Medellín. (2017, julio). Deserción en la educación superior. Boletín número 5. http://www.sapiencia.gov.co/wp-content/ uploads/2017/11/5_JULIO_BOLETIN_ODES_DESERCION_EN_LA_EDUCACION_ SUPERIOR.pdf

Olave-Arias, G., Rojas-García, I. \& Cisneros-Estupiñán, M. (2013). Deserción universitaria y alfabetización académica. Educ. Educ. 16(3), 455-471. https://www. redalyc.org/pdf/834/83429830004.pdf

Ottosen, K., Goll, C., \& Sørlie, T. (2016). The Multifaceted Challenges in Teacher-Student Relationships: A Qualitative Study of Teachers' and Principals' Experiences and Views Regarding the Dropout Rate in Norwegian Upper-Secondary Education. Scandinavian Journal of Educational Research, 1-15. https://doi.org/1 0.1080/00313831.2016.1147069

Palou, I. \& Utges, G. (2012). Teorías implícitas de docentes universitarios sobre la problemática de deserción y desgranamiento en carreras de Ingeniería. Un estudio contextualizado. Revista Argentina de Enseñanza de la Ingeniería, 1, 7-18. https://studylib.es/doc/8705707/teor\%C3\%ADas-impl\%C3\%ADcitas-de-docentes-universitarios-sobre-la-pr

Pascarella, E. T., \& Terenzini, P. T. (2005). How College Affects Students: A Third Decade of Research ( vol. 2). Jossey-Bass, An Imprint of Wiley.

Quin, D. (2016). Longitudinal and Contextual Associations Between Teacher-Student Relationships and Student Engagement: A Systematic Review. Review of Educational Research, 87(2), 1-43. https://doi.org/10.3102/0034654316669434 
Quin, D., Heerde, J. A., \& Toumbourou, J. W. (2018). Teacher support within an ecological model of adolescent development: Predictors of school engagement. Journal of School Psychology, 69, 1-15. https://10.1016/j.jsp.2018.04.003

Ryan, R., \& Deci, E. L. (2000). La Teoría de la Autodeterminación y la Facilitación de la Motivación Intrínseca, el Desarrollo Social, y el Bienestar. American Psychologist, 55(1), 68-78. https://10.1037110003-066X.55.1.68

Román, C. (2013). Factores asociados al abandono y la deserción escolar en América Latina: Una mirada en conjunto. Revista Iberoamericana sobre Calidad, Eficacia y Cambio en Educación, 11(2), 33-59. http://www.rinace.net/reice/numeros/arts/ vol11num2/art2.pdf

Rumberger, R.W. \& Rotermund, S. (2012). La relación entre el compromiso y el abandono escolar. En S.L. Christenson, A.L. Reschly y C. Wylie (Eds.). Manual de investigación sobre participación estudiantil (pp.491-513). Nueva York, NY: Springer.

Schimitt, R. E., \& Dos Santos, B. S. (2016). Permanencia estudiantil en la educación superior: un estudio metateórico basado en la bioecología del desarrollo humano. Trabajo presentado en la VI Conferencia Latinoamericana sobre el Abandono en la Educación Superior. Escuela Politécnica Nacional: Ecuador. https://revistas.utp. ac.pa/index.php/clabes/issue/view/67/65

Seidman, A. (1993). Needed: A Research Methodology to Assess Community College Effectiveness. Community College Journal, 63(5), 36-40. https://eric.ed.gov/?id=EJ461683

Silvera, L. (2016). La evaluación y su incidencia en la deserción escolar: ¿Falla de un sistema, de las instituciones educativas, del docente o del estudiante?. Educ. Humanismo, 18(31), 313-325. http://dx.doi.org/10.17081/eduhum.18.31.1381

Tejedor, F. \& García-Valcárcel, A. (2007). Causas del bajo rendimiento del estudiante universitario (en opinión de los profesores y alumnos). Propuestas de mejora en el marco del EEES. Revista de Educación, 342, 443-473. https://dialnet.unirioja. es/servlet/articulo?codigo $=2254218$

Vries, W., Arenas, P., Romero, J., \& Hernández, I. (2011). ¿Desertores o decepcionados? Distintas causas para abandonar los estudios universitarios. Revista de la educación superior, 40(160), 29-49. Recuperado de http://www.scielo.org.mx/ scielo.php?script=sci_arttext\&pid=S0185-27602011000400002

Warne, M., Svensson, A., Tirén, L. \& Wall, E. (2020). On Time: A Qualitative Study of Swedish Students', Parents' and Teachers' Views on School Attendance, with a Focus on Tardiness. International Journal of Environmental Research and Public Health, 17(1430), 1-18. https://doi.org/10.3390/ijerph17041430

Esta obra está bajo: Creative commons attribution 4.0 international license. El beneficiario de la licencia tiene el derecho de copiar distribuir, exhibir y representar la obra y hacer obras derivadas siempre y cuando reconozca y cite la obra de la forma especificada por el autor o el licenciante.

\section{(cc) BY}

